\title{
Retrograde venous bullet embolism into renal vein
}

\author{
T Pillay, ${ }^{1}$ (D) L Allopi, ${ }^{2}$ (D) HA Tariq ${ }^{3}$ \\ ${ }^{1}$ Department of Surgery, University of KwaZulu-Natal, South Africa \\ ${ }^{2}$ King Edward VII Hospital, South Africa \\ ${ }^{3}$ Prince Mshiyeni Memorial Hospital, South Africa
}

Corresponding author, email: tharugp@yahoo.co.uk

\section{Summary}

The unpredictable nature and behaviour of bullet emboli can pose unique diagnostic and management challenges, related to the absence of exit wounds or variable trajectories. However, embolisation into the vascular system is an extremely unusual occurrence, with fewer than 200 such cases described since 1900. Given the relative paucity of such literature reports, it is not surprising that guidelines for the optimal management of some of these emboli are neither clear cut, nor universally accepted. We report the second case of retrograde venous bullet embolism to the right renal vein following a gunshot injury to the right chest and the surgical solution.

Keywords: bullet embolism, renal vein, venous

\section{Case report}

A 24-year-old male presented to the casualty department approximately four hours after having sustained a gunshot injury to the right chest. Clinical evaluation confirmed a haemodynamically normal patient with a single gunshot wound to the right fifth intercostal space along the midaxillary line. Abdominal examination elicited moderate right upper quadrant tenderness on deep palpation. Plain chest radiography revealed a right-sided haemopneumothorax, which was initially managed with a closed tube thoracostomy.

A subsequent computerised tomography scan of the chest and abdomen confirmed the right-sided pleural space collection and further delineated an underlying lung contusion. Also, a metallic foreign body (bullet) was noted to be present in the region of the hilum of the right kidney.

At laparotomy, an injury to the dome of the right diaphragm was identified, together with $2 \mathrm{~cm} \times 2 \mathrm{~cm}$ gunshot wound to the left lobe of the liver (liver segment). There was no evidence of active bleeding from the liver injury, or the region surrounding the retro hepatic vena cava. The infrahepatic vena cava was intact. Inspection of the hilum of the right kidney revealed a bullet which could be visualised and palpated within a tributary of the right renal vein (Figure 1). Following trans-diaphragmatic lavage of the right pleural space and repair of the diaphragmatic injury, a decision was made to retrieve the bullet through a right renal venotomy, which was closed with a fine non-absorbable continuous suture. The right subhepatic space was drained. The patient's postoperative recovery was uneventful and remained well at six months follow-up.

\section{Discussion}

Since the first case description of a wooden missile fragment to the right heart by Thomas Davis in 1834, there have been only sporadic reports of vascular emboli in the literature. ${ }^{1}$ There was a $0.3 \%$ incidence of bullet embolism following the review of 7500 gunshot wounds that occurred during the Vietnam war. ${ }^{3}$ In a more recent review, $1.1 \%$ of 346 casualties from the Afghanistan war resulted in a bullet embolisation. ${ }^{4}$ These have largely reflected the unique challenges faced in the evolving pattern of both localisation studies and management guidelines. The presence of an incongruent number of entrance and exit wounds, foreign bodies or bullets which tend to migrate during localisation imaging studies, or bullets located in unusual sites, have not only contributed to this challenge, but may well occasionally serve a clues as to the potential likelihood of embolisation. ${ }^{3}$ There is a strong predilection for arterial embolism as opposed to venous embolism, $75 \%$ versus $25 \% .^{1,5}$ Venous embolism has been reported to largely occur in central locations with only 14 cases reporting peripheral venous embolism. ${ }^{1}$

Arterial embolisation is usually associated with acute blood loss. This coupled with the inherent risk of distal ischaemia following peripheral migration invariably necessitates immediate surgical intervention ${ }^{1}$. A review of literature has found that arterial emboli are more likely to occur on the left than the right. ${ }^{1}$ Venous bullet emboli do not usually lead to acute blood loss unless a major vessel is involved. In the majority of cases, tamponade or haemostasis prevents exsanguination. Retrograde venous bullet embolism is an extremely unusual occurrence and may be attributed to gravity, hydrostatic forces, positional changes of the patient and venous anatomy. ${ }^{6}$ The rarity of this condition may be appreciated by the fact that only 14 such cases have been described in literature. ${ }^{7}$

The majority of these venous bullet emboli are asymptomatic, up to $70 \% .^{3}$ Delayed complications occurring several years later have been well documented. These have included pulmonary vasculature emboli and the sequelae 


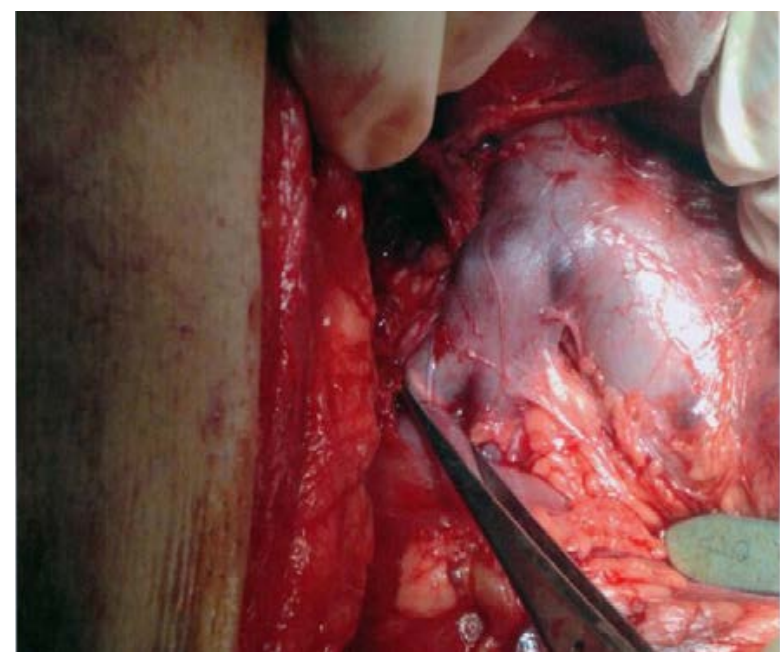

Figure 1: demonstrating bullet within the right renal vein pointed out by forceps

thereof, cardiac valvular dysfunction, dysrhythmias, bacterial endocarditis, sepsis as well as venous thrombosis. Whilst the potential benefit of retrieving asymptomatic venous emboli in preventing late complications is well documented, this should always be weighed against the potential risk of the invasive surgery associated with such retrieval. A report by Shannon et al. highlights the delayed complications of emboli and advocates mandatory acute extraction. ${ }^{1,8}$

The rationale for mandatory extraction of retrieval of venous bullet emboli, though, has traditionally been based on the risk of pulmonary embolism. The suggestion by some authors that asymptomatic bullet emboli, even within the pulmonary vasculature, may well be managed conservatively without complications has further questioned this approach. To this end, Kortbeek et al. highlighted the conservative management of pulmonary artery bullet emboli, ${ }^{9}$ whilst some authors advocate retrieval in asymptomatic patients only if an endovascular approach is feasible. ${ }^{1,10}$

It would appear that advances in minimal access and interventional radiology, together with refinement in endovascular techniques, have prompted a shift toward a more aggressive approach to retrieval, even in asymptomatic patients. These refinements have allowed for an individualised approach and management based on a riskbenefit evaluation. First reported in 1980, the endovascular technique employed a snare device for retrieval of a cardiac emboli. Regardless of the liberal use of endovascular techniques in aiding retrieval, preoperative and intraoperative imaging may help mitigate the unpredictable behaviour of the so-called "wandering bullet" emboli which have been known to migrate both prior to and even during manipulation. The use of cephalad balloon stabilisation and IVC filters can isolate a migrating bullet. ${ }^{1}$

Due to the rarity and lack of specialist experience in the field of bullet embolisation, there are a variety of approaches that can be taken with such cases. The challenge in the establishment of a general treatment algorithm is complicated by factors such as variation in anatomical locations, the timing of presentation and the presence or absence of symptoms. ${ }^{4}$

It is generally accepted that arterial emboli should be removed due to the high risk of developing end-organ damage. However, given the decreased incidence and lack of imminent life-threatening end-organ damage associated with venous emboli, there are varying opinions on its early removal. A multidisciplinary approach between interventional radiologist and vascular surgeons concluded that all intracardiac bullet emboli should be removed and conservative management can be established for select pulmonary artery emboli patients. ${ }^{4}$

Finally, the unusual path taken by the bullet in our case, involving trans-diaphragmatic entry in the liver, followed by hepatic vasculature embolisation into the vena cava, from whence it eventually lodged in the right renal vein, has not previously been described in the literature, and merits awareness. Our decision to intervene surgically and retrieve the bullet via a venotomy was based not only on the inherently unpredictable risk of long-term complications but also on the clinical presentation that warranted surgery.

\section{Conflict on interest}

The authors declare no conflict of interests.

\section{Funding source}

None.

\section{Ethics approval}

Ethics approval was received from the University of KwaZulu-Natal Biomedical Research Ethics Committee, BREC/00000471/2019.

\section{ORCID}

T Pillay (iD) https://orcid.org/0000-0002-2586-4644
L Allopi (iD $\underline{\text { https://orcid.org/0000-0002-1546-2465 }}$
HA Tariq (iD https://orcid.org/0000-0003-2790-8599

\section{REFERENCES}

1. Carter C, Havens J, Robinson W, Menard M, Gates J. Venous bullet embolism and subsequent endovascular retrieval - A case report and review of the literature. Int J Surg Case Rep. 2012;3(12):581-3. https://doi.org/10.1016/j.ijscr.2012.06.011. PMID: 22960120.

2. O'Neill P, Feldman D, Vujic I, Byrne T. Trans-jugular extraction of bullet embolus to the heart. Mil Med. 1996;161(6):360-1. PMID: 8700335.

3. Fernandez-Ranvier G, Mehta P, Zaid U, et al. Pulmonary artery bullet embolism-Case report and review. Int $\mathrm{J}$ Surg Case Rep. 2013;4(5):521-3. https://doi.org/10.1016/j. ijscr.2013.02.017. PMID: 23567547.

4. Nolan T, Phan H, Hardy A, Khanna P, Dong P. Bullet embolisation: multidisciplinary approach by interventional radiology and surgery. Semin Intervent Radiol. 2012;29(03):192-6. https://doi.org/0.1055/s-0032-1326928. PMID: 23997411.

5. Bairagi A, Hardcastle T, Muckart D. Left pulmonary artery bullet embolism following a penetrating cardiac gunshot injury. Afr J Emerg Med. 2019;9:S61-S63. https://doi. org/10.1016/j.afjem.2018.07.00. PMID: 30972288.

6. Duke E, Peterson A, Erly W. Migrating bullet: A case of a bullet embolism to the pulmonary artery with secondary pulmonary infarction after gunshot wound to the left globe. J Emerg Trauma Shock. 2014;7(1):38. https://doi.org/10.4103/09742700.125638. PMID: 24550629.

7. Bertoldo U, Enrichens F, Comba A, et al. Retrograde venous bullet embolism: a rare occurrence report and literature review. J Trauma. 2004;57(1):187-92. https://doi.org/10.1097/01. ta.0000135490.10227.5c. PMID: 15284574.

8. Shannon F, McCroskey B, Moore E, Moore F. Venous bullet embolism. J Trauma. 1987;27(10):1118-22.

9. Kortbeek J, Clark J, Carraway R. Conservative management of a pulmonary artery bullet embolism. J Trauma. 1992;33(6):906-8. https://doi.org/0.1097/00005373-1992120 00-00020. PMID: 1474637.

10. Miller K, Benns M, Sciarretta $J$, et al. The evolving management of venous bullet emboli: a case series and literature review. Injury. 2011;42(5):441-6. https://doi. org/10.1016/j.injury.2010.08.006. PMID: 20828693. 